\title{
WEAK CONVERGENCE OF AN ITERATIVE SEQUENCE FOR ACCRETIVE OPERATORS IN BANACH SPACES
}

\author{
KOJI AOYAMA, HIDEAKI IIDUKA, AND WATARU TAKAHASHI
}

Received 21 November 2005; Accepted 6 December 2005

Let $C$ be a nonempty closed convex subset of a smooth Banach space $E$ and let $A$ be an accretive operator of $C$ into $E$. We first introduce the problem of finding a point $u \in C$ such that $\langle A u, J(v-u)\rangle \geq 0$ for all $v \in C$, where $J$ is the duality mapping of $E$. Next we study a weak convergence theorem for accretive operators in Banach spaces. This theorem extends the result by Gol'shteĭn and Tret'yakov in the Euclidean space to a Banach space. And using our theorem, we consider the problem of finding a fixed point of a strictly pseudocontractive mapping in a Banach space and so on.

Copyright (C) 2006 Koji Aoyama et al. This is an open access article distributed under the Creative Commons Attribution License, which permits unrestricted use, distribution, and reproduction in any medium, provided the original work is properly cited.

\section{Introduction}

Let $H$ be a real Hilbert space with norm $\|\cdot\|$ and inner product $(\cdot, \cdot)$, let $C$ be a nonempty closed convex subset of $H$ and let $A$ be a monotone operator of $C$ into $H$. The variational inequality problem is formulated as finding a point $u \in C$ such that

$$
(v-u, A u) \geq 0
$$

for all $v \in C$. Such a point $u \in C$ is called a solution of the problem. Variational inequalities were initially studied by Stampacchia $[13,17]$ and ever since have been widely studied. The set of solutions of the variational inequality problem is denoted by $\operatorname{VI}(C, A)$. In the case when $C=H, \operatorname{VI}(H, A)=A^{-1} 0$ holds, where $A^{-1} 0=\{u \in H: A u=0\}$. An element of $A^{-1} 0$ is called a zero point of $A$. An operator $A$ of $C$ into $H$ is said to be inverse strongly monotone if there exists a positive real number $\alpha$ such that

$$
(x-y, A x-A y) \geq \alpha\|A x-A y\|^{2}
$$

for all $x, y \in C$; see Browder and Petryshyn [5], Liu and Nashed [18], and Iiduka et al. [11]. For such a case, $A$ is said to be $\alpha$-inverse strongly monotone. Let $T$ be a nonexpansive mapping of $C$ into itself. It is known that if $A=I-T$, then $A$ is $1 / 2$-inverse strongly

Hindawi Publishing Corporation

Fixed Point Theory and Applications

Volume 2006, Article ID 35390, Pages 1-13

DOI 10.1155/FPTA/2006/35390 
monotone and $F(T)=\mathrm{VI}(C, A)$, where $I$ is the identity mapping of $H$ and $F(T)$ is the set of fixed points of $T$; see [11]. In the case of $C=H=\mathbb{R}^{N}$, for finding a zero point of an inverse strongly monotone operator, Gol'shtein and Tret'yakov [8] proved the following theorem.

Theorem 1.1 (see Gol'shteln and Tret'yakov [8]). Let $\mathbb{R}^{N}$ be the $N$-dimensional Euclidean space and let $A$ be an $\alpha$-inverse strongly monotone operator of $\mathbb{R}^{N}$ into itself with $A^{-1} 0 \neq \varnothing$. Let $\left\{x_{n}\right\}$ be a sequence defined as follows: $x_{1}=x \in \mathbb{R}^{N}$ and

$$
x_{n+1}=x_{n}-\lambda_{n} A x_{n}
$$

for every $n=1,2, \ldots$, where $\left\{\lambda_{n}\right\}$ is a sequence in $[0,2 \alpha]$. If $\left\{\lambda_{n}\right\}$ is chosen so that $\lambda_{n} \in[a, b]$ for some $a, b$ with $0<a<b<2 \alpha$, then $\left\{x_{n}\right\}$ converges to some element of $A^{-1} 0$.

For finding a solution of the variational inequality for an inverse strongly monotone operator, Iiduka et al. [11] proved the following weak convergence theorem.

Theorem 1.2 (see Iiduka et al. [11]). Let $C$ be a nonempty closed convex subset of a real Hilbert space $H$ and let $A$ be an $\alpha$-inverse strongly monotone operator of $C$ into $H$ with $\operatorname{VI}(C, A) \neq \varnothing$. Let $\left\{x_{n}\right\}$ be a sequence defined as follows: $x_{1}=x \in C$ and

$$
x_{n+1}=P_{C}\left(\alpha_{n} x_{n}+\left(1-\alpha_{n}\right) P_{C}\left(x_{n}-\lambda_{n} A x_{n}\right)\right)
$$

for every $n=1,2, \ldots$, where $P_{C}$ is the metric projection from $H$ onto $C,\left\{\alpha_{n}\right\}$ is a sequence in $[-1,1]$, and $\left\{\lambda_{n}\right\}$ is a sequence in $[0,2 \alpha]$. If $\left\{\alpha_{n}\right\}$ and $\left\{\lambda_{n}\right\}$ are chosen so that $\alpha_{n} \in[a, b]$ for some $a, b$ with $-1<a<b<1$ and $\lambda_{n} \in[c, d]$ for some $c$, $d$ with $0<c<d<2(1+a) \alpha$, then $\left\{x_{n}\right\}$ converges weakly to some element of $\operatorname{VI}(C, A)$.

A mapping $T$ of $C$ into itself is said to be strictly pseudocontractive [5] if there exists $k$ with $0 \leq k<1$ such that

$$
\|T x-T y\|^{2} \leq\|x-y\|^{2}+k\|(I-T) x-(I-T) y\|^{2}
$$

for all $x, y \in C$. For such a case, $T$ is said to be $k$-strictly pseudocontractive. For finding a fixed point of a $k$-strictly pseudocontractive mapping, Browder and Petryshyn [5] proved the following weak convergence theorem.

Theorem 1.3 (Browder and Petryshyn [5]). Let $K$ be a nonempty bounded closed convex subset of a real Hilbert space $H$ and let $T$ be a $k$-strictly pseudocontractive mapping of $K$ into itself. Let $\left\{x_{n}\right\}$ be a sequence defined as follows: $x_{1}=x \in K$ and

$$
x_{n+1}=\alpha x_{n}+(1-\alpha) T x_{n}
$$

for every $n=1,2, \ldots$, where $\alpha \in(k, 1)$. Then $\left\{x_{n}\right\}$ converges weakly to some element of $F(T)$.

In this paper, motivated by the above three theorems, we first consider the following generalized variational inequality problem in a Banach space.

Problem 1.4. Let $E$ be a smooth Banach space with norm $\|\cdot\|$, let $E^{*}$ denote the dual of $E$, and let $\langle x, f\rangle$ denote the value of $f \in E^{*}$ at $x \in E$. Let $C$ be a nonempty closed convex 
subset of $E$ and let $A$ be an accretive operator of $C$ into $E$. Find a point $u \in C$ such that

$$
\langle A u, J(v-u)\rangle \geq 0, \quad \forall v \in C,
$$

where $J$ is the duality mapping of $E$ into $E^{*}$.

This problem is connected with the fixed point problem for nonlinear mappings, the problem of finding a zero point of an accretive operator and so on. For the problem of finding a zero point of an accretive operator by the proximal point algorithm, see Kamimura and Takahashi [12]. Second, in order to find a solution of Problem 1.4, we introduce the following iterative scheme for an accretive operator $A$ in a Banach space $E$ : $x_{1}=x \in C$ and

$$
x_{n+1}=\alpha_{n} x_{n}+\left(1-\alpha_{n}\right) Q_{C}\left(x_{n}-\lambda_{n} A x_{n}\right)
$$

for every $n=1,2, \ldots$, where $Q_{C}$ is a sunny nonexpansive retraction from $E$ onto $C,\left\{\alpha_{n}\right\}$ is a sequence in $[0,1]$, and $\left\{\lambda_{n}\right\}$ is a sequence of real numbers. Then we prove a weak convergence (Theorem 3.1) in a Banach space which is generalized simultaneously Gol'shtern and Tret'yakov's theorem (Theorem 1.1) and Browder and Petryshyn's theorem (Theorem $1.3)$.

\section{Preliminaries}

Let $E$ be a real Banach space with norm $\|\cdot\|$ and let $E^{*}$ denote the dual of $E$. We denote the value of $f \in E^{*}$ at $x \in E$ by $\langle x, f\rangle$. When $\left\{x_{n}\right\}$ is a sequence in $E$, we denote strong convergence of $\left\{x_{n}\right\}$ to $x \in E$ by $x_{n} \rightarrow x$ and weak convergence by $x_{n} \rightarrow x$.

Let $U=\{x \in E:\|x\|=1\}$. A Banach space $E$ is said to be uniformly convex if for each $\varepsilon \in(0,2]$, there exists $\delta>0$ such that for any $x, y \in U$,

$$
\|x-y\| \geq \varepsilon \text { implies }\left\|\frac{x+y}{2}\right\| \leq 1-\delta \text {. }
$$

It is known that a uniformly convex Banach space is reflexive and strictly convex. A Banach space $E$ is said to be smooth if the limit

$$
\lim _{t \rightarrow 0} \frac{\|x+t y\|-\|x\|}{t}
$$

exists for all $x, y \in U$. It is also said to be uniformly smooth if the limit (2.2) is attained uniformly for $x, y \in U$. The norm of $E$ is said to be Frechet differentiable if for each $x \in U$, the limit (2.2) is attained uniformly for $y \in U$. And we define a function $\rho:[0, \infty) \rightarrow$ $[0, \infty)$ called the modulus of smoothness of $E$ as follows:

$$
\rho(\tau)=\sup \left\{\frac{1}{2}(\|x+y\|+\|x-y\|)-1: x, y \in E,\|x\|=1,\|y\|=\tau\right\} .
$$

It is known that $E$ is uniformly smooth if and only if $\lim _{\tau \rightarrow 0} \rho(\tau) / \tau=0$. Let $q$ be a fixed real number with $1<q \leq 2$. Then a Banach space $E$ is said to be $q$-uniformly smooth if there exists a constant $c>0$ such that $\rho(\tau) \leq c \tau^{q}$ for all $\tau>0$. For example, see [1,23] for more details. We know the following lemma $[1,2]$. 
Lemma $2.1[1,2]$. Let $q$ be a real number with $1<q \leq 2$ and let $E$ be a Banach space. Then $E$ is $q$-uniformly smooth if and only if there exists a constant $K \geq 1$ such that

$$
\frac{1}{2}\left(\|x+y\|^{q}+\|x-y\|^{q}\right) \leq\|x\|^{q}+\|K y\|^{q}
$$

for all $x, y \in E$.

The best constant $K$ in Lemma 2.1 is called the q-uniformly smoothness constant of $E$; see [1]. Let $q$ be a given real number with $q>1$. The (generalized) duality mapping $J_{q}$ from $E$ into $2^{E^{*}}$ is defined by

$$
J_{q}(x)=\left\{x^{*} \in E^{*}:\left\langle x, x^{*}\right\rangle=\|x\|^{q},\left\|x^{*}\right\|=\|x\|^{q-1}\right\}
$$

for all $x \in E$. In particular, $J=J_{2}$ is called the normalized duality mapping. It is known that

$$
J_{q}(x)=\|x\|^{q-2} J(x)
$$

for all $x \in E$. If $E$ is a Hilbert space, then $J=I$. The normalized duality mapping $J$ has the following properties:

(1) if $E$ is smooth, then $J$ is single-valued;

(2) if $E$ is strictly convex, then $J$ is one-to-one and $\left\langle x-y, x^{*}-y^{*}\right\rangle>0$ holds for all $\left(x, x^{*}\right),\left(y, y^{*}\right) \in J$ with $x \neq y$;

(3) if $E$ is reflexive, then $J$ is surjective;

(4) if $E$ is uniformly smooth, then $J$ is uniformly norm-to-norm continuous on each bounded subset of $E$.

See [22] for more details. It is also known that

$$
q\left\langle y-x, j_{x}\right\rangle \leq\|y\|^{q}-\|x\|^{q}
$$

for all $x, y \in E$ and $j_{x} \in J_{q}(x)$. Further we know the following result [25]. For the sake of completeness, we give the proof; see also $[1,2]$.

LEMmA 2.2 [25]. Let $q$ be a given real number with $1<q \leq 2$ and let $E$ be a $q$-uniformly smooth Banach space. Then

$$
\|x+y\|^{q} \leq\|x\|^{q}+q\left\langle y, J_{q}(x)\right\rangle+2\|K y\|^{q}
$$

for all $x, y \in E$, where $J_{q}$ is the generalized duality mapping of $E$ and $K$ is the q-uniformly smoothness constant of $E$.

Proof. Let $x, y \in E$ be given arbitrarily. From (2.7), we have $q\left\langle y, J_{q}(x)\right\rangle \geq\|x\|^{q}-\| x-$ $y \|^{q}$. Thus, it follows from Lemma 2.1 that

$$
\begin{aligned}
q\left\langle y, J_{q}(x)\right\rangle & \geq\|x\|^{q}-\|x-y\|^{q} \\
& \geq\|x\|^{q}-\left(2\|x\|^{q}+2\|K y\|^{q}-\|x+y\|^{q}\right) \\
& =-\|x\|^{q}-2\|K y\|^{q}+\|x+y\|^{q} .
\end{aligned}
$$

Hence we have $\|x+y\|^{q} \leq\|x\|^{q}+q\left\langle y, J_{q}(x)\right\rangle+2\|K y\|^{q}$. 
Let $E$ be a Banach space and let $C$ be a subset of $E$. Then a mapping $T$ of $C$ into itself is said to be nonexpansive if

$$
\|T x-T y\| \leq\|x-y\|
$$

for all $x, y \in C$. We denote by $F(T)$ the set of fixed points of $T$. A closed convex subset $C$ of a Banach space $E$ is said to have normal structure if for each bounded closed convex subset $D$ of $C$ which contains at least two points, there exists an element of $D$ which is not a diametral point of $D$. It is well known that a closed convex subset of a uniformly convex Banach space has normal structure and a compact convex subset of a Banach space has normal structure. We know the following theorem [14] related to the existence of fixed points of a nonexpansive mapping.

Theorem 2.3 (Kirk [14]). Let E be a reflexive Banach space and let $D$ be a nonempty bounded closed convex subset of $E$ which has normal structure. Let $T$ be a nonexpansive mapping of $D$ into itself. Then the set $F(T)$ is nonempty.

To prove our main result, we also need the following theorem [4].

Theorem 2.4 (see Browder [4]). Let D be a nonempty bounded closed convex subset of a uniformly convex Banach space $E$ and let $T$ be a nonexpansive mapping of $D$ into itself. If $\left\{u_{j}\right\}$ is a sequence of $D$ such that $u_{j} \rightarrow u_{0}$ and $\lim _{j \rightarrow \infty}\left\|u_{j}-T u_{j}\right\|=0$, then $u_{0}$ is a fixed point of $T$. if

Let $D$ be a subset of $C$ and let $Q$ be a mapping of $C$ into $D$. Then $Q$ is said to be sunny

$$
Q(Q x+t(x-Q x))=Q x
$$

whenever $Q x+t(x-Q x) \in C$ for $x \in C$ and $t \geq 0$. A mapping $Q$ of $C$ into itself is called a retraction if $Q^{2}=Q$. If a mapping $Q$ of $C$ into itself is a retraction, then $Q z=z$ for every $z \in R(Q)$, where $R(Q)$ is the range of $Q$. A subset $D$ of $C$ is called a sunny nonexpansive retract of $C$ if there exists a sunny nonexpansive retraction from $C$ onto $D$. We know the following two lemmas $[15,20]$ concerning sunny nonexpansive retractions.

Lemma 2.5 [15]. Let $C$ be a nonempty closed convex subset of a uniformly convex and uniformly smooth Banach space $E$ and let $T$ be a nonexpansive mapping of $C$ into itself with $F(T) \neq \varnothing$. Then the set $F(T)$ is a sunny nonexpansive retract of $C$.

Lemma 2.6 (see [20]; see also [6]). Let $C$ be a nonempty closed convex subset of a smooth Banach space $E$ and let $Q_{C}$ be a retraction from $E$ onto $C$. Then the following are equivalent:

(i) $Q_{C}$ is both sunny and nonexpansive;

(ii) $\left\langle x-Q_{C} x, J\left(y-Q_{C} x\right)\right\rangle \leq 0$ for all $x \in E$ and $y \in C$.

It is well known that if $E$ is a Hilbert space, then a sunny nonexpansive retraction $Q_{C}$ is coincident with the metric projection from $E$ onto $C$. Let $C$ be a nonempty closed convex subset of a smooth Banach space $E$, let $x \in E$ and let $x_{0} \in C$. Then we have from Lemma 2.6 that $x_{0}=Q_{C} x$ if and only if $\left\langle x-x_{0}, J\left(y-x_{0}\right)\right\rangle \leq 0$ for all $y \in C$, where $Q_{C}$ is a sunny nonexpansive retraction from $E$ onto $C$. 
Let $E$ be a Banach space and let $C$ be a nonempty closed convex subset of $E$. An operator $A$ of $C$ into $E$ is said to be accretive if there exists $j(x-y) \in J(x-y)$ such that

$$
\langle A x-A y, j(x-y)\rangle \geq 0
$$

for all $x, y \in C$. We can characterize the set of solutions of Problem 1.4 by using sunny nonexpansive retractions.

Lemma 2.7. Let $C$ be a nonempty closed convex subset of a smooth Banach space E. Let $Q_{C}$ be a sunny nonexpansive retraction from $E$ onto $C$ and let $A$ be an accretive operator of $C$ into $E$. Then for all $\lambda>0$,

$$
S(C, A)=F\left(Q_{C}(I-\lambda A)\right)
$$

where $S(C, A)=\{u \in C:\langle A u, J(v-u)\rangle \geq 0, \forall v \in C\}$.

Proof. We have from Lemma 2.6 that $u \in F\left(Q_{C}(I-\lambda A)\right)$ if and only if

$$
\langle(u-\lambda A u)-u, J(y-u)\rangle \leq 0
$$

for all $y \in C$ and $\lambda>0$. This inequality is equivalent to the inequality $\langle-\lambda A u, J(y-u)\rangle \leq$ 0 . Since $\lambda>0$, we have $u \in S(C, A)$. This completes the proof.

Now, we define an extension of the inverse strongly monotone operator (1.2) in Banach spaces. Let $C$ be a subset of a smooth Banach space $E$. For $\alpha>0$, an operator $A$ of $C$ into $E$ is said to be $\alpha$-inverse strongly accretive if

$$
\langle A x-A y, J(x-y)\rangle \geq \alpha\|A x-A y\|^{2}
$$

for all $x, y \in C$. Evidently, the definition of the inverse strongly accretive operator is based on that of the inverse strongly monotone operator. It is obvious from (2.15) that

$$
\|A x-A y\| \leq \frac{1}{\alpha}\|x-y\|
$$

for all $x, y \in C$. Let $q$ be a given real number with $q \geq 2$. We also have from (2.6), (2.15), and (2.16) that

$$
\begin{aligned}
\left\langle A x-A y, J_{q}(x-y)\right\rangle & =\|x-y\|^{q-2}\langle A x-A y, J(x-y)\rangle \\
& \geq\|x-y\|^{q-2} \alpha\|A x-A y\|^{2} \\
& \geq(\alpha\|A x-A y\|)^{q-2} \alpha\|A x-A y\|^{2} \\
& =\alpha^{q-1}\|A x-A y\|^{q}
\end{aligned}
$$

for all $x, y \in C$. One should note that no Banach space is $q$-uniformly smooth for $q>2$; see [23] for more details. So, in this paper, we study a weak convergence theorem for inverse strongly accretive operators in uniformly convex and 2-uniformly smooth Banach spaces. It is well known that Hilbert spaces and the Lebesgue $L^{p}(p \geq 2)$ spaces are 
uniformly convex and 2-uniformly smooth. Let $X$ be a Banach space and let $L^{p}(X)=$ $L^{p}(\Omega, \Sigma, \mu ; X), 1 \leq p \leq \infty$, be the Lebesgue-Bochner space on an arbitrary measure space $(\Omega, \Sigma, \mu)$. Let $1<q \leq 2$ and let $q \leq p<\infty$. Then $L^{p}(X)$ is $q$-uniformly smooth if and only if $X$ is $q$-uniformly smooth; see [23]. For convergence theorems in the Lebesgue spaces $L^{p}(1<p \leq 2)$, see Iiduka and Takahashi $[9,10]$.

We can know the following property for inverse strongly accretive operators in a 2uniformly smooth Banach space.

LEMMA 2.8. Let $C$ be a nonempty closed convex subset of a 2-uniformly smooth Banach space E. Let $\alpha>0$ and let $A$ be an $\alpha$-inverse strongly accretive operator of $C$ into $E$. If $0<\lambda \leq \alpha / K^{2}$, then $I-\lambda A$ is a nonexpansive mapping of $C$ into $E$, where $K$ is the 2-uniformly smoothness constant of $E$.

Proof. We have from Lemma 2.2 that for all $x, y \in C$,

$$
\begin{aligned}
\|(I-\lambda A) x-(I-\lambda A) y\|^{2} & =\|(x-y)-\lambda(A x-A y)\|^{2} \\
& \leq\|x-y\|^{2}-2 \lambda\langle A x-A y, J(x-y)\rangle+2 K^{2} \lambda^{2}\|A x-A y\|^{2} \\
& \leq\|x-y\|^{2}-2 \lambda \alpha\|A x-A y\|^{2}+2 K^{2} \lambda^{2}\|A x-A y\|^{2} \\
& \leq\|x-y\|^{2}+2 \lambda\left(K^{2} \lambda-\alpha\right)\|A x-A y\|^{2} .
\end{aligned}
$$

So, if $0<\lambda \leq \alpha / K^{2}$, then $I-\lambda A$ is a nonexpansive mapping of $C$ into $E$.

Remark 2.9. If $q \geq 2$, we have from (2.17) that for $x, y \in C$,

$$
\|(I-\lambda A) x-(I-\lambda A) y\|^{q} \leq\|x-y\|^{q}+\lambda\left(2 K^{q} \lambda^{q-1}-q \alpha^{q-1}\right)\|A x-A y\|^{q} .
$$

Since, for $q>2$, there exists no Banach space which is $q$-uniformly smooth, we consider only 2 -uniformly smooth Banach spaces. For $1<q<2$, the inequalities (2.17) and (2.19) do not hold.

Applying Theorem 2.3, Lemmas 2.7 and 2.8, we have that if $D$ is a nonempty bounded closed convex subset of a uniformly convex and 2-uniformly smooth Banach space $E, D$ is a sunny nonexpansive retract of $E$ and $A$ is an inverse strongly accretive operator of $D$ into $E$, then the set $S(D, A)$ is nonempty. We know also the following theorem which was proved by Reich [21]; see also Lau and Takahashi [16], Takahashi and Kim [24], and Bruck [7].

Theorem 2.10 (see Reich [21]). Let $C$ be a nonempty closed convex subset of a uniformly convex Banach space with a Frechet differentiable norm. Let $\left\{T_{1}, T_{2}, \ldots\right\}$ be a sequence of nonexpansive mappings of $C$ into itself with $\bigcap_{n=1}^{\infty} F\left(T_{n}\right) \neq \varnothing$. Let $x \in C$ and $S_{n}=T_{n} T_{n-1} \cdots T_{1}$ for all $n \geq 1$. Then the set

$$
\bigcap_{n=1}^{\infty} \overline{c o}\left\{S_{m} x: m \geq n\right\} \cap \bigcap_{n=1}^{\infty} F\left(T_{n}\right)
$$

consists of at most one point, where $\overline{c o} D$ is the closure of the convex hull of $D$. 


\section{Weak convergence theorem}

In this section, we obtain the following weak convergence theorem for finding a solution of Problem 1.4 for an inverse strongly accretive operator in a uniformly convex and 2uniformly smooth Banach space.

Theorem 3.1. Let E be a uniformly convex and 2-uniformly smooth Banach space and let $C$ be a nonempty closed convex subset of $E$. Let $Q_{C}$ be a sunny nonexpansive retraction from $E$ onto $C$, let $\alpha>0$ and let $A$ be an $\alpha$-inverse strongly accretive operator of $C$ into $E$ with $S(C, A) \neq \varnothing$. Suppose $x_{1}=x \in C$ and $\left\{x_{n}\right\}$ is given by

$$
x_{n+1}=\alpha_{n} x_{n}+\left(1-\alpha_{n}\right) Q_{C}\left(x_{n}-\lambda_{n} A x_{n}\right)
$$

for every $n=1,2, \ldots$, where $\left\{\lambda_{n}\right\}$ is a sequence of positive real numbers and $\left\{\alpha_{n}\right\}$ is a sequence in $[0,1]$. If $\left\{\lambda_{n}\right\}$ and $\left\{\alpha_{n}\right\}$ are chosen so that $\lambda_{n} \in\left[a, \alpha / K^{2}\right]$ for some $a>0$ and $\alpha_{n} \in[b, c]$ for some $b, c$ with $0<b<c<1$, then $\left\{x_{n}\right\}$ converges weakly to some element $z$ of $S(C, A)$, where $K$ is the 2-uniformly smoothness constant of $E$.

Proof. Put $y_{n}=Q_{C}\left(x_{n}-\lambda_{n} A x_{n}\right)$ for every $n=1,2, \ldots$ Let $u \in S(C, A)$. We first prove that $\left\{x_{n}\right\}$ and $\left\{y_{n}\right\}$ are bounded and $\lim _{n \rightarrow \infty}\left\|x_{n}-y_{n}\right\|=0$. We have from Lemmas 2.7 and 2.8 that

$$
\begin{aligned}
\left\|y_{n}-u\right\| & =\left\|Q_{C}\left(x_{n}-\lambda_{n} A x_{n}\right)-Q_{C}\left(u-\lambda_{n} A u\right)\right\| \\
& \leq\left\|\left(x_{n}-\lambda_{n} A x_{n}\right)-\left(u-\lambda_{n} A u\right)\right\| \leq\left\|x_{n}-u\right\|
\end{aligned}
$$

for every $n=1,2, \ldots$. It follows from (3.2) that

$$
\begin{aligned}
\left\|x_{n+1}-u\right\| & =\left\|\alpha_{n}\left(x_{n}-u\right)+\left(1-\alpha_{n}\right)\left(y_{n}-u\right)\right\| \\
& \leq \alpha_{n}\left\|x_{n}-u\right\|+\left(1-\alpha_{n}\right)\left\|y_{n}-u\right\| \\
& \leq \alpha_{n}\left\|x_{n}-u\right\|+\left(1-\alpha_{n}\right)\left\|x_{n}-u\right\|=\left\|x_{n}-u\right\|
\end{aligned}
$$

for every $n=1,2, \ldots$. Therefore, $\left\{\left\|x_{n}-u\right\|\right\}$ is nonincreasing and hence there exists $\lim _{n \rightarrow \infty}\left\|x_{n}-u\right\|$. So, $\left\{x_{n}\right\}$ is bounded. We also have from (3.2) and (2.16) that $\left\{y_{n}\right\}$ and $\left\{A x_{n}\right\}$ are bounded.

Next we will show $\lim _{n \rightarrow \infty}\left\|x_{n}-y_{n}\right\|=0$. Suppose that $\lim _{n \rightarrow \infty}\left\|x_{n}-y_{n}\right\| \neq 0$. Then there are $\varepsilon>0$ and a subsequence $\left\{x_{n_{i}}-y_{n_{i}}\right\}$ of $\left\{x_{n}-y_{n}\right\}$ such that $\left\|x_{n_{i}}-y_{n_{i}}\right\| \geq \varepsilon$ for each $i=1,2, \ldots$. Since $E$ is uniformly convex, the function $\|\cdot\|^{2}$ is uniformly convex on bounded convex set $B\left(0,\left\|x_{1}-u\right\|\right)$, where $B\left(0,\left\|x_{1}-u\right\|\right)=\left\{x \in E:\|x\| \leq\left\|x_{1}-u\right\|\right\}$. So, for $\varepsilon$, there is $\delta>0$ such that

$$
\|x-y\| \geq \varepsilon \text { implies }\|\lambda x+(1-\lambda) y\|^{2} \leq \lambda\|x\|^{2}+(1-\lambda)\|y\|^{2}-\lambda(1-\lambda) \delta
$$

whenever $x, y \in B\left(0,\left\|x_{1}-u\right\|\right)$ and $\lambda \in(0,1)$. Thus, for each $i=1,2, \ldots$,

$$
\begin{aligned}
\left\|x_{n_{i}+1}-u\right\|^{2} & =\left\|\alpha_{n_{i}}\left(x_{n_{i}}-u\right)+\left(1-\alpha_{n_{i}}\right)\left(y_{n_{i}}-u\right)\right\|^{2} \\
& \leq \alpha_{n_{i}}\left\|x_{n_{i}}-u\right\|^{2}+\left(1-\alpha_{n_{i}}\right)\left\|y_{n_{i}}-u\right\|^{2}-\alpha_{n_{i}}\left(1-\alpha_{n_{i}}\right) \delta .
\end{aligned}
$$


Therefore, for each $i=1,2, \ldots$,

$$
0<b(1-c) \delta \leq \alpha_{n_{i}}\left(1-\alpha_{n_{i}}\right) \delta \leq\left\|x_{n_{i}}-u\right\|^{2}-\left\|x_{n_{i}+1}-u\right\|^{2} .
$$

Since the right-hand side of the inequality above converges to 0 , we have a contradiction. Hence we conclude that

$$
\lim _{n \rightarrow \infty}\left\|x_{n}-y_{n}\right\|=0 \text {. }
$$

Since $\left\{x_{n}\right\}$ is bounded, we have that a subsequence $\left\{x_{n_{i}}\right\}$ of $\left\{x_{n}\right\}$ converges weakly to $z$. And since $\lambda_{n_{i}}$ is in $\left[a, \alpha / K^{2}\right]$ for some $a>0$, it holds that $\left\{\lambda_{n_{i}}\right\}$ is bounded. So, there exists a subsequence $\left\{\lambda_{n_{i j}}\right\}$ of $\left\{\lambda_{n_{i}}\right\}$ which converges to $\lambda_{0} \in\left[a, \alpha / K^{2}\right]$. We may assume without loss of generality that $\lambda_{n_{i}} \rightarrow \lambda_{0}$. We next prove $z \in S(C, A)$. Since $Q_{C}$ is nonexpansive, it holds from $y_{n_{i}}=Q_{C}\left(x_{n_{i}}-\lambda_{n_{i}} A x_{n_{i}}\right)$ that

$$
\begin{aligned}
\left\|Q_{C}\left(x_{n_{i}}-\lambda_{0} A x_{n_{i}}\right)-x_{n_{i}}\right\| & \leq\left\|Q_{C}\left(x_{n_{i}}-\lambda_{0} A x_{n_{i}}\right)-y_{n_{i}}\right\|+\left\|y_{n_{i}}-x_{n_{i}}\right\| \\
& \leq\left\|\left(x_{n_{i}}-\lambda_{0} A x_{n_{i}}\right)-\left(x_{n_{i}}-\lambda_{n_{i}} A x_{n_{i}}\right)\right\|+\left\|y_{n_{i}}-x_{n_{i}}\right\| \\
& \leq M\left|\lambda_{n_{i}}-\lambda_{0}\right|+\left\|y_{n_{i}}-x_{n_{i}}\right\|,
\end{aligned}
$$

where $M=\sup \left\{\left\|A x_{n}\right\|: n=1,2, \ldots\right\}$. We obtain from the convergence of $\left\{\lambda_{n_{i}}\right\},(3.7)$, and (3.8) that

$$
\lim _{i \rightarrow \infty}\left\|Q_{C}\left(I-\lambda_{0} A\right) x_{n_{i}}-x_{n_{i}}\right\|=0 .
$$

On the other hand, from Lemma 2.8, we have that $Q_{C}\left(I-\lambda_{0} A\right)$ is nonexpansive. So, by (3.9), Lemma 2.7, and Theorem 2.4, we obtain $z \in F\left(Q_{C}\left(I-\lambda_{0} A\right)\right)=S(C, A)$.

Finally, we prove that $\left\{x_{n}\right\}$ converges weakly to some element of $S(C, A)$. We put

$$
T_{n}=\alpha_{n} I+\left(1-\alpha_{n}\right) Q_{C}\left(I-\lambda_{n} A\right)
$$

for every $n=1,2, \ldots$. Then we have $x_{n+1}=T_{n} T_{n-1} \cdots T_{1} x$ and $z \in \bigcap_{n=1}^{\infty} \overline{c o}\left\{x_{m}: m \geq n\right\}$. We have from Lemma 2.8 that $T_{n}$ is a nonexpansive mapping of $C$ into itself for every $n=1,2, \ldots$. And we also have from Lemma 2.7 that $\bigcap_{n=1}^{\infty} F\left(T_{n}\right)=\bigcap_{n=1}^{\infty} F\left(Q_{C}\left(I-\lambda_{n} A\right)\right)=$ $S(C, A)$. Applying Theorem 2.10, we obtain

$$
\bigcap_{n=1}^{\infty} \overline{c o}\left\{x_{m}: m \geq n\right\} \cap S(C, A)=\{z\} .
$$

Therefore, the sequence $\left\{x_{n}\right\}$ converges weakly to some element of $S(C, A)$. This completes the proof.

\section{Applications}

In this section, we prove some weak convergence theorems in a uniformly convex and 2-uniformly smooth Banach space by using Theorem 3.1. We first study the problem of finding a zero point of an inverse strongly accretive operator. The following theorem is a generalization of Gol'shteĭn and Tret'yakov's theorem (Theorem 1.1). 
Theorem 4.1. Let E be a uniformly convex and 2-uniformly smooth Banach space. Let $\alpha>0$ and let $A$ be an $\alpha$-inverse strongly accretive operator of $E$ into itself with $A^{-1} 0 \neq \varnothing$, where $A^{-1} 0=\{u \in E: A u=0\}$. Suppose $x_{1}=x \in E$ and $\left\{x_{n}\right\}$ is given by

$$
x_{n+1}=x_{n}-r_{n} A x_{n}
$$

for every $n=1,2, \ldots$, where $\left\{r_{n}\right\}$ is a sequence of positive real numbers. If $\left\{r_{n}\right\}$ is chosen so that $r_{n} \in[s, t]$ for some $s, t$ with $0<s<t<\alpha / K^{2}$, then $\left\{x_{n}\right\}$ converges weakly to some element $z$ of $A^{-1} 0$, where $K$ is the 2-uniformly smoothness constant of $E$.

Proof. By assumption, we note that $1-t K^{2} / \alpha \in(0,1)$. We define sequences $\left\{\alpha_{n}\right\}$ and $\left\{\lambda_{n}\right\}$ by

$$
\alpha_{n}=1-t \frac{K^{2}}{\alpha}, \quad \lambda_{n}=\frac{r_{n}}{1-\alpha_{n}}
$$

for every $n=1,2, \ldots$, respectively. Then it is easy to check that $\lambda_{n} \in\left(0, \alpha / K^{2}\right)$ and $S(E$, $A)=A^{-1} 0$. It follows from the definition of $\left\{x_{n}\right\}$ that

$$
\begin{aligned}
x_{n+1} & =x_{n}-r_{n} A x_{n}=\alpha_{n} x_{n}+\left(1-\alpha_{n}\right)\left(x_{n}-\frac{r_{n}}{1-\alpha_{n}} A x_{n}\right) \\
& =\alpha_{n} x_{n}+\left(1-\alpha_{n}\right) I\left(x_{n}-\lambda_{n} A x_{n}\right),
\end{aligned}
$$

where $I$ is the identity mapping of $E$. Obviously, the identity mapping $I$ is a sunny nonexpansive retraction from $E$ onto itself. Therefore, by using Theorem 3.1, $\left\{x_{n}\right\}$ converges weakly to some element $z$ of $A^{-1} 0$.

We next study the problem of finding a fixed point of a strictly pseudocontractive mapping. Let $0 \leq k<1$. Let $E$ be a Banach space and let $C$ be a subset of $E$. Then a mapping $T$ of $C$ into itself is said to be $k$-strictly pseudocontractive $[5,19]$ if there exists $j(x-y) \in J(x-y)$ such that

$$
\langle T x-T y, j(x-y)\rangle \leq\|x-y\|^{2}-\frac{1-k}{2}\|(I-T) x-(I-T) y\|^{2}
$$

for all $x, y \in C$. Then the inequality (4.4) can be written in the form

$$
\langle(I-T) x-(I-T) y, j(x-y)\rangle \geq \frac{1-k}{2}\|(I-T) x-(I-T) y\|^{2} .
$$

If $E$ is a Hilbert space, then the inequality (4.4) (and hence (4.5)) is equivalent to the inequality (1.5). The following theorem is a generalization of Browder and Petryshyn's theorem (Theorem 1.3).

Theorem 4.2. Let E be a uniformly convex and 2-uniformly smooth Banach space and let $C$ be a nonempty closed convex subset and a sunny nonexpansive retract of $E$. Let $T$ be a $k$-strictly pseudocontractive mapping of $C$ into itself with $F(T) \neq \varnothing$. Suppose $x_{1}=x \in C$ and $\left\{x_{n}\right\}$ is given by

$$
x_{n+1}=\left(1-\beta_{n}\right) x_{n}+\beta_{n} T x_{n}
$$


for every $n=1,2, \ldots$, where $\left\{\beta_{n}\right\}$ is a sequence in $(0,1)$. If $\left\{\beta_{n}\right\}$ is chosen so that $\beta_{n} \in[\beta, \gamma]$ for some $\beta, \gamma$ with $0<\beta<\gamma<(1-k) /\left(2 K^{2}\right)$, then $\left\{x_{n}\right\}$ converges weakly to some element $z$ of $F(T)$, where $K$ is the 2-uniformly smoothness constant of $E$.

Proof. By assumption, note that $1-2 \gamma K^{2} /(1-k) \in(0,1)$. We define sequences $\left\{\alpha_{n}\right\}$ and $\left\{\lambda_{n}\right\}$ by

$$
\alpha_{n}=1-\gamma \frac{2 K^{2}}{1-k}, \quad \lambda_{n}=\frac{\beta_{n}}{1-\alpha_{n}}
$$

for every $n=1,2, \ldots$, respectively. Then we can readily verify that

$$
0<\lambda_{n} \leq \frac{1-k}{2 K^{2}} \leq \frac{1}{2}<1
$$

for every $n=1,2, \ldots$. Put $A=I-T$. We have from (4.5) that $A$ is $(1-k) / 2$-inverse strongly accretive. It is easy to show that

$$
S(C, A)=S(C, I-T)=F(T) \neq \varnothing .
$$

Since $C$ is a sunny nonexpansive retract of $E$ and $\lambda_{n} \in(0,1)$, there exists a sunny nonexpansive retraction $Q_{C}$ such that $\left(1-\lambda_{n}\right) x_{n}+\lambda_{n} T x_{n}=Q_{C}\left(\left(1-\lambda_{n}\right) x_{n}+\lambda_{n} T x_{n}\right)$ for every $n=1,2, \ldots$ It follows from the definition of $\left\{x_{n}\right\}$ that

$$
\begin{aligned}
x_{n+1} & =\left(1-\beta_{n}\right) x_{n}+\beta_{n} T x_{n} \\
& =\left(1-\lambda_{n}\left(1-\alpha_{n}\right)\right) x_{n}+\lambda_{n}\left(1-\alpha_{n}\right) T x_{n} \\
& =\alpha_{n} x_{n}+\left(1-\alpha_{n}\right)\left(\left(1-\lambda_{n}\right) x_{n}+\lambda_{n} T x_{n}\right) \\
& =\alpha_{n} x_{n}+\left(1-\alpha_{n}\right) Q_{C}\left(\left(1-\lambda_{n}\right) x_{n}+\lambda_{n} T x_{n}\right) \\
& =\alpha_{n} x_{n}+\left(1-\alpha_{n}\right) Q_{C}\left(x_{n}-\lambda_{n}(I-T) x_{n}\right) \\
& =\alpha_{n} x_{n}+\left(1-\alpha_{n}\right) Q_{C}\left(x_{n}-\lambda_{n} A x_{n}\right) .
\end{aligned}
$$

Therefore, by using Theorem 3.1, $\left\{x_{n}\right\}$ converges weakly to some element $z$ of $F(T)$.

Let $C$ be a subset of a smooth Banach space $E$. Let $\alpha>0$. An operator $A$ of $C$ into $E$ is said to be $\alpha$-strongly accretive if

$$
\langle A x-A y, J(x-y)\rangle \geq \alpha\|x-y\|^{2}
$$

for all $x, y \in C$. Let $\beta>0$. An operator $A$ of $C$ into $E$ is said to be $\beta$-Lipschitz continuous if

$$
\|A x-A y\| \leq \beta\|x-y\|
$$

for all $x, y \in C$. Let $C$ be a nonempty closed convex subset of a Hilbert space $H$. One method of finding a point $u \in \operatorname{VI}(C, A)$ is the projection algorithm which starts with any $x_{1}=x \in C$ and updates iteratively $x_{n+1}$ according to the formula

$$
x_{n+1}=P_{C}\left(x_{n}-\lambda A x_{n}\right)
$$


for every $n=1,2, \ldots$, where $P_{C}$ is the metric projection from $H$ onto $C, A$ is a monotone (accretive) operator of $C$ into $H$, and $\lambda$ is a positive real number. It is well known that if $A$ is an $\alpha$-strongly accretive and $\beta$-Lipschitz continuous operator of $C$ into $H$ and $\lambda \in\left(0,2 \alpha / \beta^{2}\right)$, then the operator $P_{C}(I-\lambda A)$ is a contraction of $C$ into itself. Hence, the Banach contraction principle guarantees that the sequence generated by $(4.13)$ converges strongly to the unique solution of $\operatorname{VI}(C, A)$; see [3]. Motivated by this result, we prove the following weak convergence theorem for strongly accretive and Lipschitz continuous operators.

THeOREM 4.3. Let E be a uniformly convex and 2-uniformly smooth Banach space and let $C$ be a nonempty closed convex subset of $E$. Let $Q_{C}$ be a sunny nonexpansive retraction from $E$ onto $C$, let $\alpha>0$, let $\beta>0$, and let $A$ be an $\alpha$-strongly accretive and $\beta$-Lipschitz continuous operator of $C$ into $E$ with $S(C, A) \neq \varnothing$. Suppose $x_{1}=x \in C$ and $\left\{x_{n}\right\}$ is given by

$$
x_{n+1}=\alpha_{n} x_{n}+\left(1-\alpha_{n}\right) Q_{C}\left(x_{n}-\lambda_{n} A x_{n}\right)
$$

for every $n=1,2, \ldots$, where $\left\{\lambda_{n}\right\}$ is a sequence of positive real numbers and $\left\{\alpha_{n}\right\}$ is a sequence in $[0,1]$. If $\left\{\lambda_{n}\right\}$ and $\left\{\alpha_{n}\right\}$ are chosen so that $\lambda_{n} \in\left[a, \alpha /\left(K^{2} \beta^{2}\right)\right]$ for some $a>0$ and $\alpha_{n} \in$ $[b, c]$ for some $b, c$ with $0<b<c<1$, then $\left\{x_{n}\right\}$ converges weakly to a unique element $z$ of $S(C, A)$, where $K$ is the 2-uniformly smoothness constant of $E$.

Proof. Since $A$ is an $\alpha$-strongly accretive and $\beta$-Lipschitz continuous operator of $C$ into $E$, we have

$$
\langle A x-A y, J(x-y)\rangle \geq \alpha\|x-y\|^{2} \geq \frac{\alpha}{\beta^{2}}\|A x-A y\|^{2}
$$

for all $x, y \in C$. Therefore, $A$ is $\alpha / \beta^{2}$-inverse strongly accretive. Since $A$ is strongly accretive and $S(C, A) \neq \varnothing$, the set $S(C, A)$ consists of one point $z$. Using Theorem 3.1, $\left\{x_{n}\right\}$ converges weakly to a unique element $z$ of $S(C, A)$.

\section{References}

[1] K. Ball, E. A. Carlen, and E. H. Lieb, Sharp uniform convexity and smoothness inequalities for trace norms, Inventiones Mathematicae 115 (1994), no. 3, 463-482.

[2] B. Beauzamy, Introduction to Banach Spaces and Their Geometry, 2nd ed., North-Holland Mathematics Studies, vol. 68, North-Holland, Amsterdam, 1985.

[3] H. Brezis, Analyse Fonctionnelle. Théorie et Applications, Collection of Applied Mathematics for the Master's Degree, Masson, Paris, 1983.

[4] F. E. Browder, Nonlinear operators and nonlinear equations of evolution in Banach spaces, Nonlinear Functional Analysis (Proc. Sympos. Pure Math., Vol. XVIII, Part 2, Chicago, Ill, 1968), American Mathematical Society, Rhode Island, 1976, pp. 1-308.

[5] F. E. Browder and W. V. Petryshyn, Construction of fixed points of nonlinear mappings in Hilbert space, Journal of Mathematical Analysis and Applications 20 (1967), 197-228.

[6] R. E. Bruck Jr., Nonexpansive retracts of Banach spaces, Bulletin of the American Mathematical Society 76 (1970), 384-386.

[7] A simple proof of the mean ergodic theorem for nonlinear contractions in Banach spaces, Israel Journal of Mathematics 32 (1979), no. 2-3, 107-116.

[8] E. G. Gol'shtern and N. V. Tret'yakov, Modified Lagrangians in convex programming and their generalizations, Mathematical Programming Study (1979), no. 10, 86-97. 
[9] H. Iiduka and W. Takahashi, Strong convergence of a projection algorithm by hybrid type for monotone variational inequalities in a Banach space, in preparation.

[10] Weak convergence of a projection algorithm for variational inequalities in a Banach space, in preparation.

[11] H. Iiduka, W. Takahashi, and M. Toyoda, Approximation of solutions of variational inequalities for monotone mappings, Panamerican Mathematical Journal 14 (2004), no. 2, 49-61.

[12] S. Kamimura and W. Takahashi, Weak and strong convergence of solutions to accretive operator inclusions and applications, Set-Valued Analysis 8 (2000), no. 4, 361-374.

[13] D. Kinderlehrer and G. Stampacchia, An Introduction to Variational Inequalities and Their Applications, Pure and Applied Mathematics, vol. 88, Academic Press, New York, 1980.

[14] W. A. Kirk, A fixed point theorem for mappings which do not increase distances, The American Mathematical Monthly 72 (1965), 1004-1006.

[15] S. Kitahara and W. Takahashi, Image recovery by convex combinations of sunny nonexpansive retractions, Topological Methods in Nonlinear Analysis 2 (1993), no. 2, 333-342.

[16] A. T. Lau and W. Takahashi, Weak convergence and nonlinear ergodic theorems for reversible semigroups of nonexpansive mappings, Pacific Journal of Mathematics 126 (1987), no. 2, 277-294.

[17] J.-L. Lions and G. Stampacchia, Variational inequalities, Communications on Pure and Applied Mathematics 20 (1967), 493-519.

[18] F. Liu and M. Z. Nashed, Regularization of nonlinear ill-posed variational inequalities and convergence rates, Set-Valued Analysis 6 (1998), no. 4, 313-344.

[19] M. O. Osilike and A. Udomene, Demiclosedness principle and convergence theorems for strictly pseudocontractive mappings of Browder-Petryshyn type, Journal of Mathematical Analysis and Applications 256 (2001), no. 2, 431-445.

[20] S. Reich, Asymptotic behavior of contractions in Banach spaces, Journal of Mathematical Analysis and Applications 44 (1973), no. 1, 57-70.

[21] W Weak convergence theorems for nonexpansive mappings in Banach spaces, Journal of Mathematical Analysis and Applications 67 (1979), no. 2, 274-276.

[22] W. Takahashi, Nonlinear Functional Analysis. Fixed Point Theory and Its Applications, Yokohama Publishers, Yokohama, 2000.

[23] Y. Takahashi, K. Hashimoto, and M. Kato, On sharp uniform convexity, smoothness, and strong type, cotype inequalities, Journal of Nonlinear and Convex Analysis 3 (2002), no. 2, 267-281.

[24] W. Takahashi and G.-E. Kim, Approximating fixed points of nonexpansive mappings in Banach spaces, Mathematica Japonica 48 (1998), no. 1, 1-9.

[25] H. K. Xu, Inequalities in Banach spaces with applications, Nonlinear Analysis 16 (1991), no. 12, $1127-1138$.

Koji Aoyama: Department of Economics, Chiba University, Yayoi-Cho, Inage-Ku,

Chiba-Shi, Chiba 263-8522, Japan

E-mail address: aoyama@le.chiba-u.ac.jp

Hideaki Iiduka: Department of Mathematical and Computing Sciences,

Tokyo Institute of Technology, Oh-Okayama, Meguro-Ku, Tokyo 152-8522, Japan

E-mail address: hideaki.iiduka@is.titech.ac.jp

Wataru Takahashi: Department of Mathematical and Computing Sciences,

Tokyo Institute of Technology, Oh-Okayama, Meguro-Ku, Tokyo 152-8522, Japan

E-mail address: wataru@is.titech.ac.jp 